\title{
The Light Weight Design of Mowing Vehicle Frame with a Combined Method
}

\author{
Shunming Li*, Kun Xu, Jinrui Wang \\ College of Energy and Power Engineering, Nanjing University of Aeronautics and Astronautics, Nanjing, China \\ Email: ^smli@nuaa.edu.cn,691735562@qq.com,240588630@qq.com
}

How to cite this paper: Li, S.M., Xu, K. and Wang, J.R. (2018) The Light Weight Design of Mowing Vehicle Frame with a Combined Method. Modern Mechanical Engineering, 8, 192-203.

https://doi.org/10.4236/mme.2018.83013

Received: April 20, 2018

Accepted: July 14, 2018

Published: July 17, 2018

Copyright $\odot 2018$ by authors and Scientific Research Publishing Inc. This work is licensed under the Creative Commons Attribution International License (CC BY 4.0).

http://creativecommons.org/licenses/by/4.0/

\begin{abstract}
Lightweight frame is very important to engineering machinery. In this paper, a lightweight design method is proposed for a mechanical mowing truck frame. This method combines topological optimization with topology optimization to design the frame successfully. Based on the finite element simulation, the strength analysis of the two working conditions (bending condition and torsion condition) for the mowing vehicle frame is carried out on the basis of satisfying the requirements of the frame work strength. This paper makes a comparative analysis of the frame after the second optimization using the combined method proposed. The comparison results show that the optimized frame meets the strength requirement, and its quality is $34.3 \%$ lower than before. The lightweight effect is obvious.
\end{abstract}

\section{Keywords}

Lightweight, Finite Element Simulation, Optimization, Work Strength

\section{Introduction}

Energy conservation and environmental protection are always the theme of world development. On a global scale, the total energy is limited. And with the rapid development of economy, energy consumption is also increasing. Foreign relevant research data indicate that the fuel consumption can be reduced by $6 \%$ $8 \%$ for every $10 \%$ reduction in vehicle weight [1]. Lightweight research can reduce energy consumption, reduce the manufacturing and use costs, improve the economic benefit, so it is very meaningful to study lightweight [2] [3] [4]. There are many domestic and foreign scholars studied the lightweight design.

The paper optimizes the motorcycle frame, making its minimum quality, and the lightweight effect is remarkable [5]. The paper optimizes the structural parts 
with steel as raw material, and effectively reduces the quality [6] [7] [8]. In this paper [9] [10], the method of size parameter optimization for frame is designed, and lightweight effect is obvious. Size optimization is usually optimized for unit size [11] [12], which is easy to achieve the optimization goal. But it is difficult to improve the original structure. Topology optimization [13] can find the optimal topology by constraint load conditions. This method compared with the size optimization is much more difficulty, but this method can be changing the original structure, and this is its outstanding feature. Mower frame, as the base body of the main parts of the vehicle, bears the force of gravity and the force between the parts and the frame.

The reliability of the frame directly affects the operation of the vehicle and the safety of the driver. Therefore, the lightweight design should be carried out under the condition that the intensity is met. The mower frame has both beam structure and slab structure. There is room for improvement in overall structure and unit size. So this paper combines the advantages of topology optimization and size optimization. Topology optimization is carried out first, and dimensional optimization is carried out on the basis of topology optimization. Finally, the required frame structure is obtained, and compared with the original frame. We examine the advantages and disadvantages of the optimization results and whether the results are more satisfactory than the single optimization.

\section{The Establish of Original Model for Mowing Frame}

Owing to its function, the mowing vehicle is different from the traditional passenger car. There is a big difference in the form of the frame. The whole frame is composed of 27 parts, such as the front plate of the baffle battery seat and the front and rear support beams. The frame model is built with Solid works software, and we simplify the unnecessary parts. The remaining parts are modeled according to the actual size. Each component is bound according to the actual situation. After the completion of the assembly model is shown in Figure 1.

\section{Frame Strength Analysis}

Steel copper and aluminum and other plastic materials usually fail in the form of submission. In finite element analysis, the static strength of materials can be

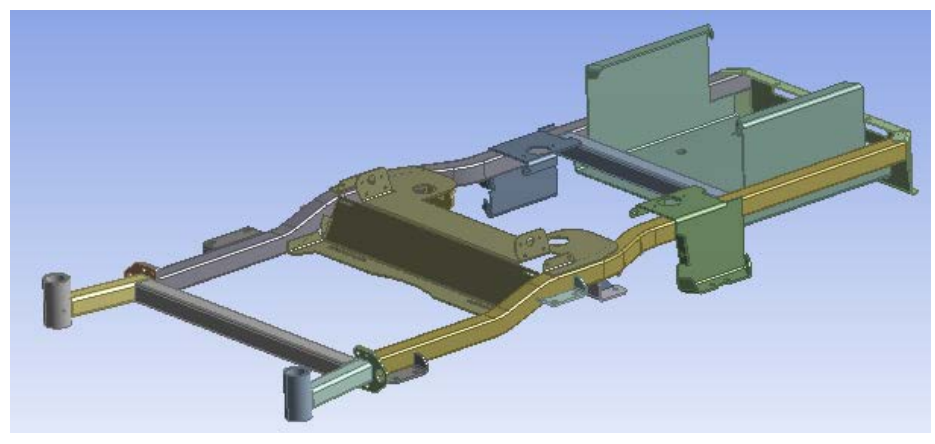

Figure 1. Original model of mowing vehicle frame. 
judged by the size of the equivalent stress of Von Mises. The frame material is Q235 steel. Yield strength is $\sigma_{s}=235 \mathrm{MPa}$. Elasticity modulus is $E=210 \mathrm{GPa}$. Poisson's ratio is $v=0.3$. The maximum stress is not exceeding the allowable stress of the material. The effect force can be expressed as.

$$
\sigma_{r}=\sqrt{\frac{1}{2}\left[\left(\sigma_{1}-\sigma_{2}\right)^{2}+\left(\sigma_{2}-\sigma_{3}\right)^{2}+\left(\sigma_{3}-\sigma_{1}\right)^{2}\right]}
$$

The condition of meeting strength should be established: $\sigma_{r} \leq[\sigma]$. There into, $\sigma_{r}$ is Equivalent stress, $\sigma_{1}, \sigma_{2}, \sigma_{3}$ respectively represent the first and second and third principal stresses. $[\sigma]$ indicates the allowable stress of the material. And $\sigma_{s} /[\sigma]=n, n$ is safety factor.

Because of the different load, the frame can produce different deformation in the process of work. And bending deformation and torsional deformation is the most common deformation, corresponding bending working condition and twisting working condition, which are the two kinds of typical working condition. These two kinds of typical working conditions are the most basic conditions, and it is also the two important conditions that have a great affect on frame. Therefore, this article selects bending condition and twisting working condition which are the two kinds of typical working condition to check the frame strength.

Using the finite element simulation, we import the model established in Solid works for static analysis. The load and constraints of the frame are applied in accordance with the actual conditions. The main force of the frame is the force on the frame, the force of the driver on the frame, the force of the rear battery pack on the frame, and the weight of the frame. The driver's quality is $90 \mathrm{~kg}$, and the knife dish quality is $98 \mathrm{~kg}$. Finally, quality of battery pack is $80 \mathrm{~kg}$.

\subsection{Bending Condition}

Under bending condition, the constraint condition is the four supporting points of fixed constraint of the whole frame. All parts of the frame are bound to each other. The specific load and constraints are shown in Figure 2 (the parts A-M stand for the force of the main nodes respectively). The results of stress can be obtained by calculation. The equivalent stress of Von Miss is shown in Figure 3.

It can be seen from Figure 3 that the maximum equivalent stress of the whole frame is $39.5 \mathrm{MPa}$, and the rest area stress is not more than $20 \mathrm{MPa}$, and the safety factor is selected as 3 . Allowable stress $[\sigma]=78.3 \mathrm{MPa}$, so the maximum equivalent stress $\sigma_{r} \leq[\sigma]$. Therefore, in the bending condition, the larger safety factor is selected, the frame strength meets the requirements, and there is still a large margin, so there is a large optimization space.

\subsection{Working Condition of Reverse}

In the torsion condition, the degree of freedom of a front-end supporting vertical direction is released, and the other three supports are fixed. All parts of the frame are bound to each other. The specific load and constraints are shown in 


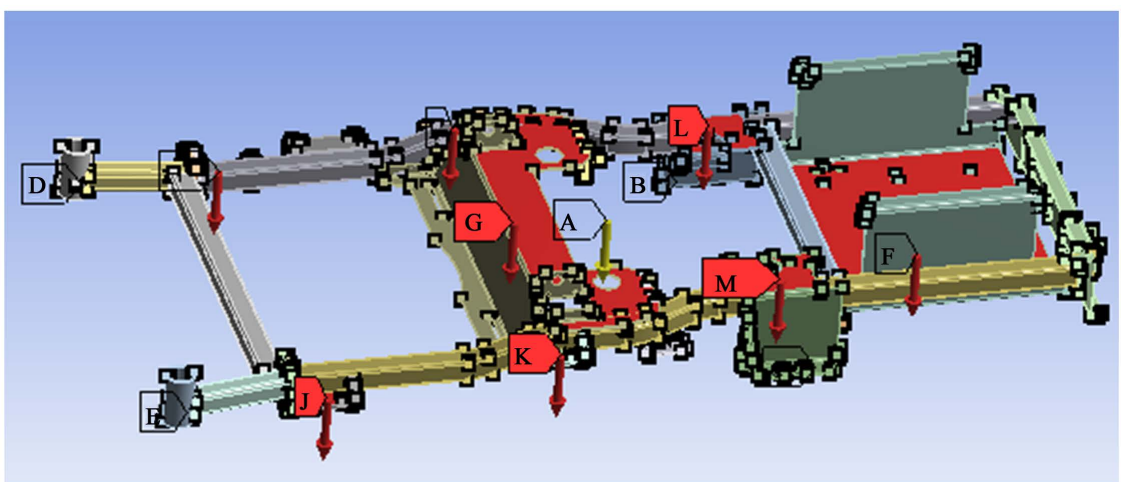

Figure 2. Loading and restraint of bending conditions.

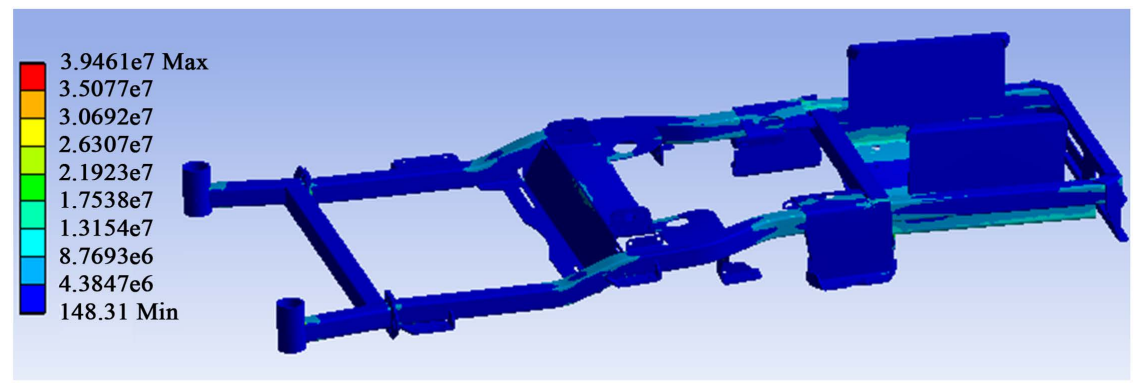

Figure 3. The equivalent stress cloud diagram of the Von Mises in the bending condition.

Figure 4 (the parts A-M stand for the force of the main nodes respectively). The results of stress can be obtained by calculation. Von Miss equivalent stress is shown in Figure 5.

As can be seen from Figure 5, the maximum equivalent stress of the whole frame is $53.1 \mathrm{MPa}$, and the rest of the stress is not more than $20 \mathrm{MPa}$. The safety factor is selected 3. So the allowable stress is $[\sigma]=78.3 \mathrm{MPa}$. Therefore, the maximum equivalent stress $\sigma_{r} \leq[\sigma]$. And in the torsional condition, the larger safety factor is selected, the frame strength meets the requirements, and there is still a large margin, so there is a large optimization space.

\section{Frame Optimization Design}

It can be seen from the analysis of two typical working conditions that the overall stress of the frame is small, and the frame strength has a large margin. There is a lot of waste in the utilization of materials, so it is necessary to carry out lightweight design. The object characteristics of topology optimization and size optimization are different. It is used for different occasions. The mower frame has both beam structure and slab structure, and there is room for improvement in overall structure and unit size. As a result, this paper adopts topology optimization and size optimization of two-stage optimization method to optimize design, in order to achieve better weight loss.

\subsection{Basic Concept of Topology Optimization}

The purpose of topology optimization design is to find the optimal distribution 


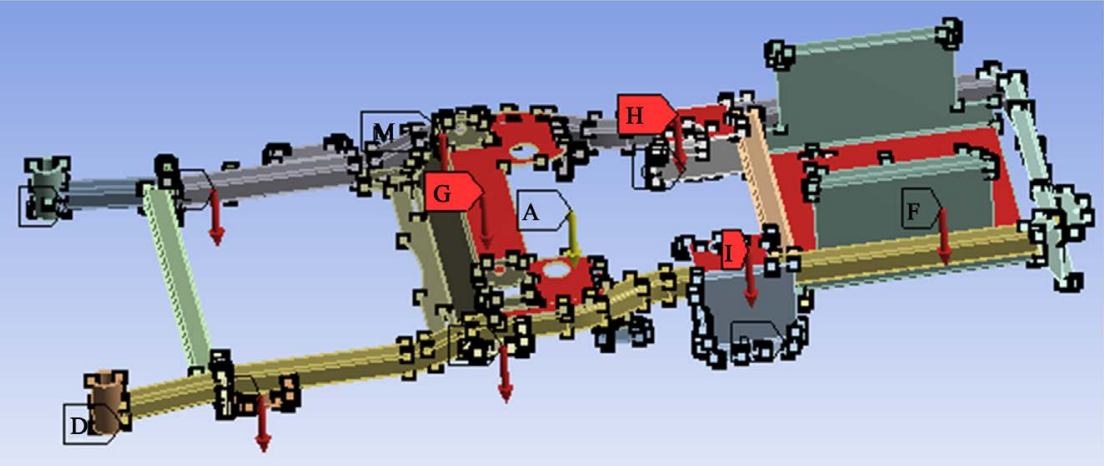

Figure 4. Load and restraint of torsional conditions.

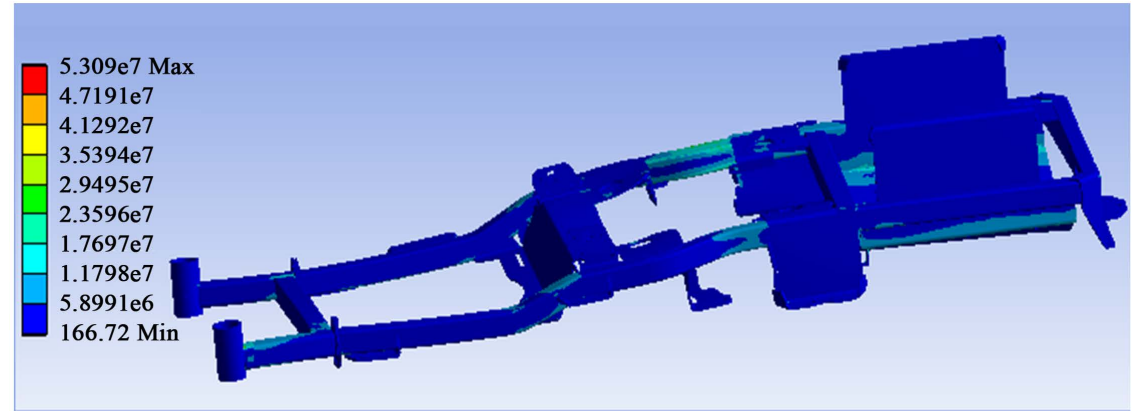

Figure 5. The equivalent stress cloud of the Von Mises in the torsional condition.

of shapes and materials within the specified design space, commonly used in concept design phase. The basic design idea of structural topology optimization is to transform the optimal topological structure of the search structure into the problem of finding the optimal material distribution in a given design area. In product concept design phase, it is not enough to through experience to design the structure. Only by analyzing the topology optimization design and combining with the rich design experience of the designer, can the reasonable scheme be obtained in the early stage of conceptual design. Finally, the design products meet the requirements. The essence of continuum topology optimization is the combination of 0 - 1 discrete variables, and the variable density method is commonly used.

In the process of topology optimization, the unit density is selected as the design variable, and the mathematical model can be written.

$$
\begin{gathered}
\text { Minimize: } f(x)=f\left(x_{1}, x_{2}, \cdots, x_{n}\right) \\
\text { Constraint condition } \begin{cases}g_{j}(x) \leq 0 & j=1, \cdots, m \\
h_{k}(x)=0 & k=1, \cdots, h \\
x_{i}^{L} \leq x_{i} \leq x_{i}^{U} & i=1, \cdots, n\end{cases}
\end{gathered}
$$

There into, $X=x_{1}, x_{2}, \cdots, x_{n}$ is the design variable; $f(x)$ is the target function; $g(X)$ is inequality constraint function; $h(X)$ is equality constraint function; $x_{i}$ is design variable; $L$ is lower limit; $U$ is upper limit. 


\subsection{Size Optimization Basic Concept}

Size optimization design is a target without changing topological structure under the premise of geometric shape and material properties, relying on dimension parameters as design variables, through specific mathematical model of the optimization algorithm to optimize the parameters of the structure to minimize the mass or volume. The size optimization design is used in the detailed design stage. It can meet certain design requirements (such as stress frequency displacement, etc.) by changing the properties of structural elements (such as the thickness of shell elements, pole of cross-sectional area, etc.). So using the method of finite element theory to calculate the structural displacement and stress, it does not change the target topology structure size parameters, such as attributes. It can directly optimize the existing structure size parameters, reduce the cost and improve the structure performance.

In the process of dimension optimization, the thickness of each part is selected as the design variable, and the mathematical model can be expressed as:

$$
\begin{aligned}
& \text { Minimize: } M=\rho \sum_{i=1}^{n} v_{i} \\
& \text { Constraint condition: }\left\{\begin{array}{l}
\sigma_{\max } \leq \sigma_{e} \\
x_{i}^{L} \leq x_{i} \leq x_{i}^{U} \quad i=1, \cdots, n
\end{array}\right.
\end{aligned}
$$

There into, $M$ is the overall quality of frame structure; is the material density; $v_{i}$ is the element volume; $n$ is the number of units; $\sigma_{\max }$ is the maximum stress value of element in finite element analysis; $\sigma_{e}$ is the material yield limit, $x_{i}$ is the design variable; $L$ is lower limit; $U$ is upper limit.

The lightweight design scheme of the frame is shown in Figure 6.

In this paper, the bending and torsion conditions are respectively selected to

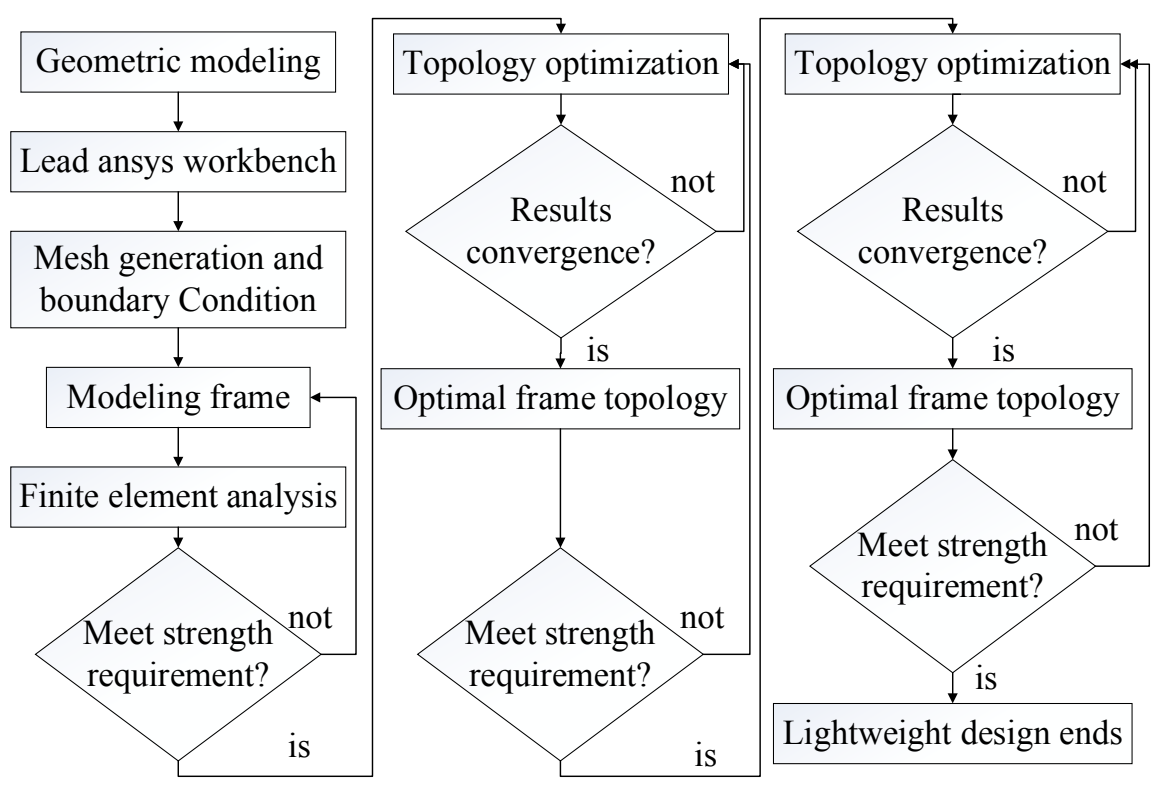

Figure 6. The process of lightweight design scheme of frame. 
optimize the topology, and the two conditions are basically same. The specific steps are as follows:

1) In the two working conditions, the static analysis of the frame was carried out. The frame model was imported into the workbench, and the material properties, load and constraints were set in the static analysis module, and the static force was solved after the network completed.

2) After the static analysis, we insert shape optimization module after static analysis module and enter the module for loading and restraint. The load applied is the same as the constraint and static analysis. We set a $30 \%$ reduction in target reduction. After the setting, the topology optimization is solved.

3) The model was modified according to the topological optimization results, and the modified model was then analyzed to verify whether the modified results met the requirements.

The overall topology optimization results are shown in Figure 7. In Figure 7, the red area is the part that can theoretically be removed. However, when the model is actually modified, the relative rule is usually removed according to the actual situation. In theory, the red zone can be completely removed, but in fact some red areas may be associated with some of the parts that have been simplified, so sometimes they cannot be completely removed.

In the whole frame, the main parts are left and right long beam, front and rear cross beams, battery seat, rear baffle and front plate. So the main goal of topology optimization is to select these components. According to Figure 7, the topology modification of these parts is carried out. The left and right baffles of the battery are equal to the left and right beam; the upper and lower edges of the rear baffle are excavated; the rear beam is excavated at the front; the front of the beam and the back are excavated; the back end of the left and right beam is excavated; the front panel does not have topological modifications. After modification, the figures of each part are shown in Figure 8.
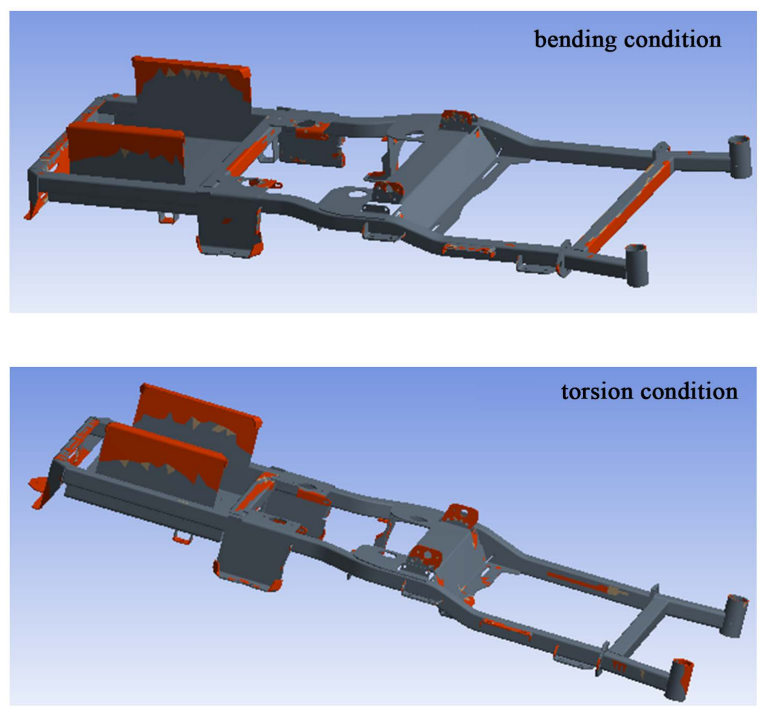

Figure 7. The overall topology optimization results. 


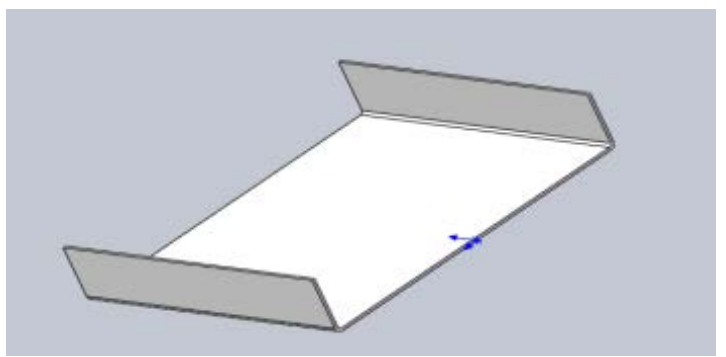

(a)

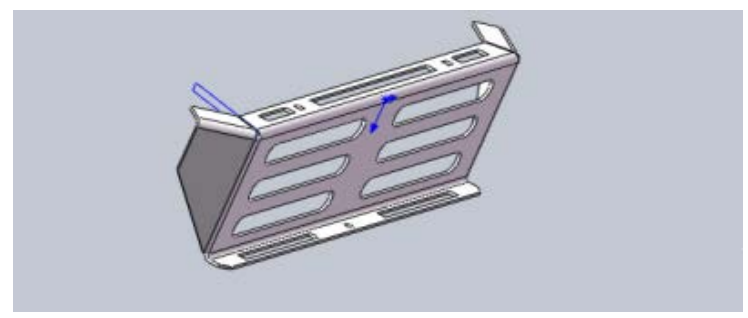

(b)

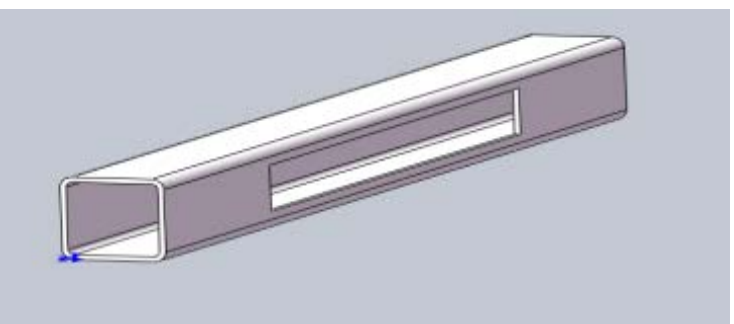

(c)

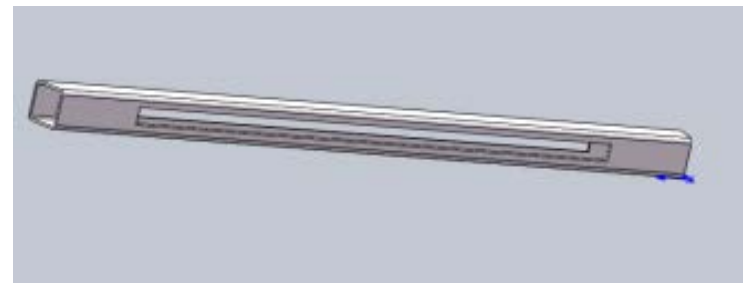

(d)

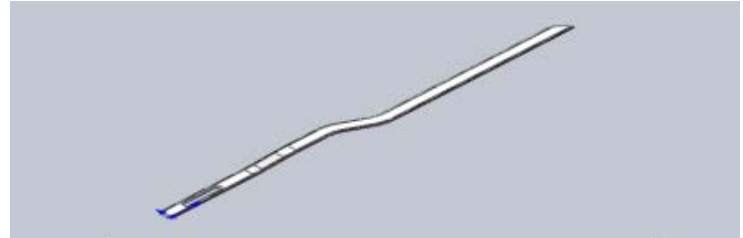

(e)

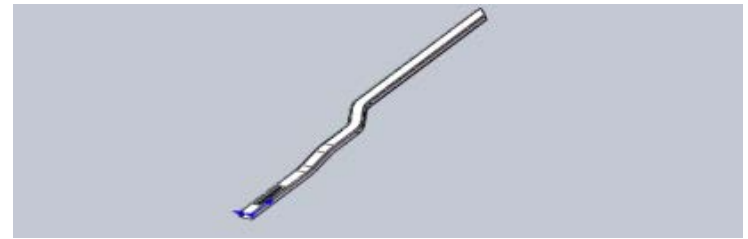

(f)

Figure 8. The topology modification results of the main parts. (a) Battery seat; (b) Rear baffle; (c) Rear cross beams; (d) Front cross beams; (e) Right long beam; (f) Left long beam. 
According to the result of modification, the strength of bending and torsion condition is rectified, and the calculation results are shown in Figure 9.

It can be seen from Figure 9 that the maximum stress of the two working conditions after modification is $43 \mathrm{MPa}$ and $44 \mathrm{MPa}$, while the rest of the stress is not more than $20 \mathrm{MPa}$. In this paper, the safety factor is selected as 3 . Allowable stress is $[\sigma]=78.3 \mathrm{MPa}$. So the maximum equivalent stress is $\sigma_{r} \leq[\sigma]$, and this meet the intensity requirements. Overall frame of the original weighs $67.075 \mathrm{~kg}$. Through the topology optimization, the overall frame weights 59.69 $\mathrm{kg}, 7.385 \mathrm{~kg}$ reduction, reduced $11 \%$. A good weight loss effect is achieved. However, most of the overall frame stress is still small, so there is still room for further optimization.

On the basis of topology optimization, we select the thickness of the large plate, the thickness of the battery seat, the thickness of the left and right beam, the thickness of the front beam, the thickness of the back beam, and the seven dimension parameters of the rear baffle as the design variables. The constraint conditions are selected as maximum equivalent stress without exceeding allowable stress. The constraint conditions are selected as maximum equivalent stress without exceeding allowable stress. The finite element simulation is used to optimize the target drive of bending and torsion.

The finite element simulation use the Design Explorer as the optimization tool, and this tool studies the output parameters through the design point parameters. But input design point is limited in general, so the response surface is usually studied by a finite number of design points. According to the selected 7

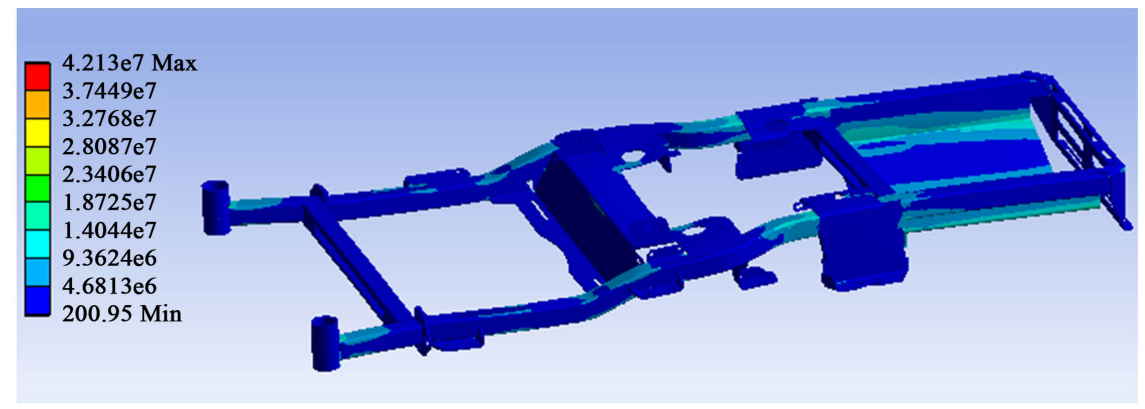

(a)

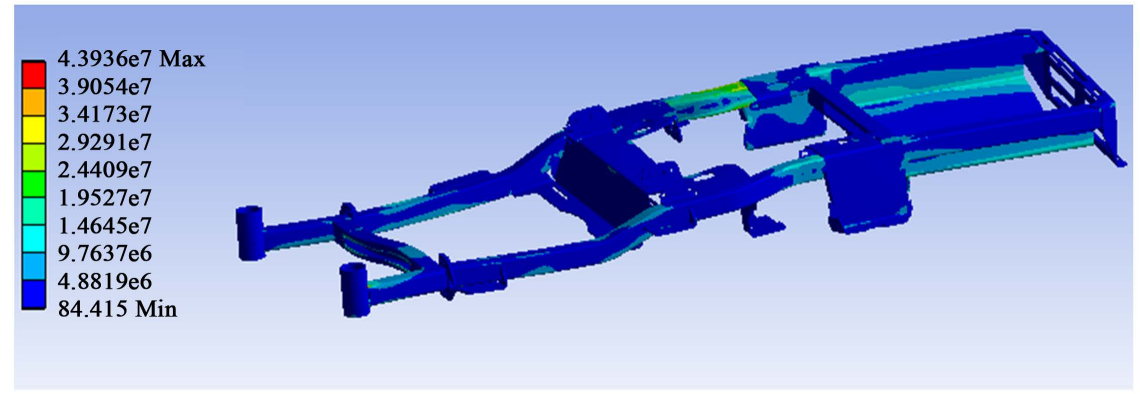

(b)

Figure 9. The Von Mises equivalent stress cloud diagram. (a) Bending condition; (b) Torsion condition. 
size parameters, 79 design points are generated by the software. By calculation, in both cases, recommended optimal design points are generated according to the set constraints and target functions. The two design points are inserted into the original finite element model for static calculation. The selection and calculation results of dimension values are shown in Table 1.

According to the calculation results of two working conditions, we select the size values that can satisfy the requirements of both conditions. The final selection is shown in Table 2. Next, we check the strength. The effect force of the calculation can be obtained in two working conditions as shown in Figure 10.

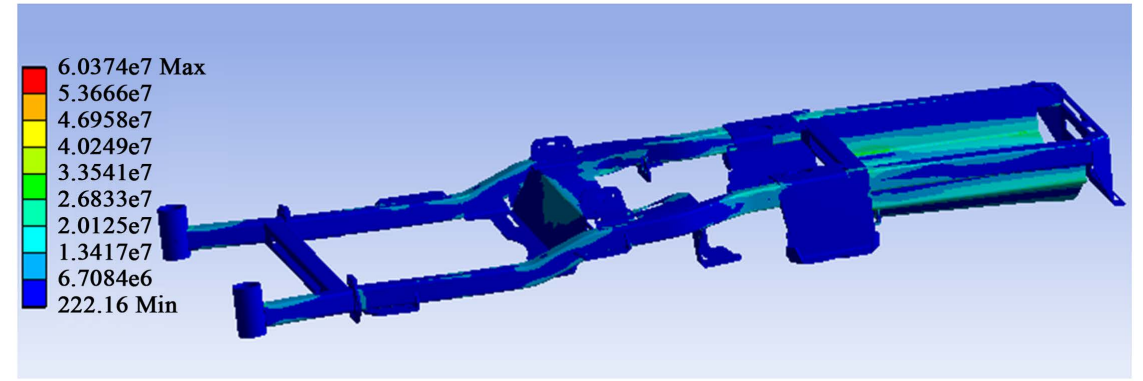

(a)

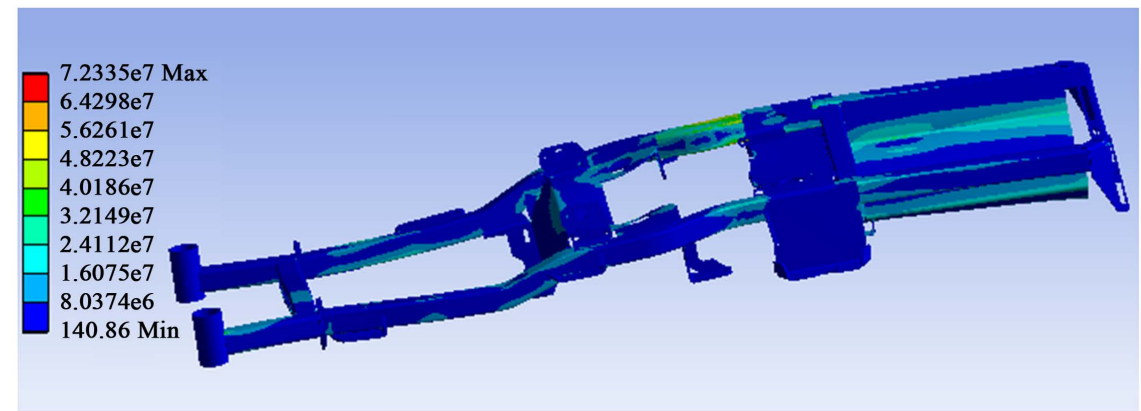

(b)

Figure 10. The Von Mises equivalent stress cloud diagram. (a) Bending condition; (b) Torsion condition.

Table 1. The objective driven optimization results.

\begin{tabular}{ccc}
\hline & Bending condition & Torsion condition \\
\hline Big board & $2.421 \mathrm{~mm}$ & $2.113 \mathrm{~mm}$ \\
Battery seat & $3.3451 \mathrm{~mm}$ & $3.6637 \mathrm{~mm}$ \\
Left long beam & $1.7374 \mathrm{~mm}$ & $1.6991 \mathrm{~mm}$ \\
Right long beam & $1.5795 \mathrm{~mm}$ & $1.5357 \mathrm{~mm}$ \\
Front cross beams & $1.6592 \mathrm{~mm}$ & $1.8848 \mathrm{~mm}$ \\
Rear cross beams & $1.8094 \mathrm{~mm}$ & $2.5378 \mathrm{~mm}$ \\
Rear baffle & $2.7284 \mathrm{~mm}$ & $2.3978 \mathrm{~mm}$ \\
Maximum Equivalent Stress & $69.97 \mathrm{MPa}$ & $42.356 \mathrm{~kg}$ \\
Overall quality of the frame & $42.356 \mathrm{~kg}$ & $42.898 \mathrm{~kg}$ \\
\hline
\end{tabular}


Table 2. The size optimization results.

\begin{tabular}{cc}
\hline Component thickness & Unit (mm) \\
\hline Big board & 2.4 \\
Battery seat & 3.7 \\
Left long beam & 1.7 \\
Right long beam & 1.7 \\
Front cross beams & 1.9 \\
Rear cross beams & 2.6 \\
Rear baffle & 2.4 \\
\hline
\end{tabular}

It can be seen from Figure 10 that, under the bending condition, the maximum equivalent stress of the Von Mises modified model is $60.37 \mathrm{MPa}$. In the case of the safety factor 3 , it is less than allowable stress $[\sigma]=78.3 \mathrm{MPa}$, so this satisfies the intensity requirement. Under the condition of torsion, the equivalent stress of Von Mises modified model is maximal 72.34 MPa. In the case of the safety factor 3 , it is less than allowable stress $[\sigma]=78.3 \mathrm{MPa}$, so it also meets the intensity requirements. After the optimization of topological optimization and size optimization, the frame quality was reduced to $44.053 \mathrm{~kg}$. The original mass was $67.075 \mathrm{~kg}$, and the weight was reduced by $23.022 \mathrm{~kg}$, which decreased by $34.3 \%$, and it achieved a good light quantification effect.

\section{Conclusions}

The following conclusions are drawn from the above analysis:

1) In this paper, Solid works software is used to model the parameterization of mowing frame. The strength analysis of bending condition and torsion working condition was conducted by the finite element simulation. The maximum equivalent stress of the two conditions is $39.5 \mathrm{MPa}$ and $53.1 \mathrm{MPa}$ respectively. The stress in most areas is not more than $20 \mathrm{MPa}$, which is less than the allowable stress of 78.3 MPa. This meets the intensity requirements. The overall frame has a lot of optimization space.

2) Topology optimization of frame is carried out. The maximum stress in the two conditions of the optimized frame is $43 \mathrm{MPa}$ and $44 \mathrm{MPa}$, which is less than the allowable stress of 78.3 MPa. This meets the intensity requirements. There is still room for further refinement. The overall frame was reduced from $67.075 \mathrm{~kg}$ to $59.69 \mathrm{~kg}$, which decreased by $7.385 \mathrm{~kg}$ and decreased by $11 \%$. We've got a good weight loss effect initially.

3) Further dimensional optimization was carried out. The maximum stress is 60.37 $\mathrm{MPa}$ and $72.34 \mathrm{MPa}$ in the two conditions of the optimized frame, which is less than the allowable stress of 78.3 MPa. This meets the intensity requirements. The overall frame weight was reduced from $59.69 \mathrm{~kg}$ to $44.053 \mathrm{~kg}$ and $15.637 \mathrm{~kg}$. A total of $23.022 \mathrm{~kg}$ was alleviated through two-stage optimization, and $34.3 \%$ weight was finally reduced. The lightweight design effect is remarkable. 


\section{Acknowledgements}

This work was supported by National Key Research and Development Program of China (2016YFD0700800).

\section{References}

[1] Hudson, S.W. and Apelian, D. (2016) Inclusion Detection in Molten Aluminum: Current Art and New Avenues for In Situ Analysis. International Journal of Metalcasting, 10, 289-305. https://doi.org/10.1007/s40962-016-0030-x

[2] Han, H., Chen, S., Shao, J., et al. (2013) Lightweight Design of Chassis Frame for Motor Boom Sprayer. Transactions of the Chinese Society of Agricultural Engineering, 29, 47-53.

[3] Feng, Q., Yu, S., Luo, J., et al. (2011) Research on Lightweight Design of Vice-Frame Structure of Fire-Engine on Basis of ANSYS. International Conference on Computer-Aided Industrial Design \& Conceptual Design, Yiwu, 17-19 November 2010, 1347-1350.

[4] Mao, P.H., Zhai, B. and Li, J.X. (2013) The Lightweight Design for Tractor Frame of the Coal Mine Belt Horizontal Extension Transfer Machine. Advanced Materials Research, 791-793, 730-733.

https://doi.org/10.4028/www.scientific.net/AMR.791-793.730

[5] Matsumoto, M., Abe, J. and Oda, J. (1991) A Structural Optimization for Configuration Design of the Motorcycle Body.

[6] Yoshitake, A. (2011) Ultralight Steel Auto Body-Advanced Vehicle Concept. Journal of the Japan Welding Society.

[7] Grüner, M. and Merklein, M. (2014) Determination of Friction Coefficients in Deep Drawing by Modification of Siebel's Formula for Calculation of Ideal Drawing Force. Production Engineering, 8, 577-584.

https://doi.org/10.1007/s11740-014-0551-1

[8] Wei, C., Zhang, J., Yang, S., et al. (2015) Experiment-Based Regional Characterization of HAZ Mechanical Properties for Laser Welding. International Journal of Advanced Manufacturing Technology, 78, 1629-1640. https://doi.org/10.1007/s00170-014-6762-y

[9] Yuan, Q., Zhou, G. and Weidong, H.E. (2013) Structure Design and Parameter Optimization of Lightweight Composite Canister Cover. Hangkong Xuebao/acta Aeronautica Et Astronautica Sinica, 34, 826-832.

[10] Ju, S., Shenoi, R.A., Jiang, D., et al. (2013) Multi-Parameter Optimization of Lightweight Composite Triangular Truss Structure Based on Response Surface Methodology. Composite Structures, 97, 107-116.

https://doi.org/10.1016/j.compstruct.2012.10.025

[11] Ramakrishnan, K. (2014) Title Optimization and Process Modelling of Municipal Solid Waste using Plasma Gasification for Power Generation in Trichy, India.

[12] Jahani, A., Feghhi, J., Makhdoum, M.F., et al. (2016) Optimized Forest Degradation Model (OFDM): An Environmental Decision Support System for Environmental Impact Assessment Using an Artificial Neural Network. Journal of Environmental Planning \& Management, 59, 222-244. https://doi.org/10.1080/09640568.2015.1005732

[13] Paulino, G.H. (2016) Bridging Topology Optimization and Additive Manufacturing. Springer-Verlag, New York. 